\title{
Split ICSI/Insemination in Mild Male Factor Infertility: a Randomized Controlled Trial
}

\author{
Ashraf Moawad, a,c Hanaa Abou-Ria, a,d Mohamed Abd Elzaher , a Mohamed \\ Farahat, ${ }^{b}$ and Mahmoud Shaeer, ${ }^{c}$ \\ ${ }^{a}$ Assistant Professor of Obstetrics and Gynecology (Al-Azhar University, Cairo, Egypt), ${ }^{\text {b }}$ \\ Lecturer of Obstetrics and Gynecology (Al-Azhar University, Cairo, Egypt), ${ }^{\mathrm{c}}$ Enjab \\ Hospital for infertility (Sharjah, UAE) and ${ }^{\mathrm{d}}$ Gulf Medical College and Research Centre
} (Ajman, UAE).

\begin{abstract}
Introduction: Intracytoplasmic sperm injection (ICSI) is a widely used adjunct to in vitro fertilization (IVF) for male-factor infertility when fertilization failure is suspected. In patients with borderline semen, the decision to choose either IVF or ICSI is critical because the chance of total fertilization failure after a conventional IVF or of performing an unnecessary ICSI procedure is hard to predict.
\end{abstract}

Objective: The purpose of this study was to assess the value of the allocation of some eggs to ICSI and others to conventional insemination (Split ICSI/insemination) in IVF cycles in which semen parameters are subfertile.

Design: It is prospective study.

Materials and methods: Between August 2007 and April 2010, 190 patients had half of their eggs inseminated with ICSI and the other half inseminated with conventional IVF.

Results: In this study, $76.3 \%$ of patients were fertilized by both IVF and ICSI, $21.6 \%$ of patients were fertilized only by ICSI while $2.1 \%$ of patients were not fertilized either by IVF or ICSI. Fertilization rate was significantly higher in oocytes injected by ICSI $(63.7 \%)$ compared to oocyte inseminated by IVF $(44.3 \%)$. In patients fertilized by both IVF and ICSI, although ICSI fertilization rate was higher $(65.9 \%)$ than IVF fertilization rate $(57.3 \%)$, yet there is no significant differences were observed. High grade embryos were significantly higher in oocytes injected by ICSI $(75.9 \%)$ compared to oocyte inseminated by IVF $(56.4 \%)$. Also, significantly more type 1 and 2 embryos developed after ICSI compared with IVF $(77.3 \%$ vs. 56.4\%) in the group of patients with fertilization after both IVF and ICSI.

Conclusion: According to our findings, implementation of ICSI in couples with mild male factor infertility could improve fertilization rates and decrease the risk of complete fertilization failure. Additionally, split ICSI procedure provides valuable clinical information about fertilization potential for the couple and unnecessary use of ICSI procedure can be avoided in future cycles for patients who have achieved good fertilization in both IVF and ICSI.

Keywords: ICSI(Intracytoplsmic sperm injection)-IVF(Invitrofertlization)-OCCs(Oocytecumulus complexes). 


\section{Introduction}

In assisted reproduction programmes, decisions concerning the treatment technique (IVF or ICSI) are usually made after the evaluation of male fertility factors, or taking into account the results of previous IVF attempts. There are no widely accepted criteria, so decisions for couples with male subfertility (i.e. at least one sperm characteristic abnormal) are often empirical and may lead to complete fertilization failure after IVF, or to the unnecessary use of ICSI.

Threshold values of sperm parameters for assisted procreation are based mainly on the World Health Organization standard (1999) and widely are used to discriminate between male fertility and subfertility (Pisarska1999, Verheyen G 1999 \& HlagerhA2002). However, the prognostic value of those parameters is questionable (LiuDY2000, LiuDY2002 \& GuzickDS2001). Mahutte and Arici (2003) conducted a review of different screening tests. Their conclusion was that more sophisticated methods such as sperm-zona binding ratios and zona pellucida-induced acrosome reaction tests may improve the ability to predict fertilization capacity, but unfortunately, no test can exclude the possibility of fertilization failure.

The majority of failed fertilized oocytes do not contain sperm nuclei after conventional IVF (WallMB1996 \& Edirisingh1997) indicating that most cases of fertilization failure relate to an inability of the sperm cell to penetrate the oocyte. Oocyte-related factors that might account for fertilization failure in some cases could be defects in the pronuclear formation or an oocyte activation failure (MahutteNG2003).

Intracytoplasmic sperm injection (ICSI) with its high fertilization and pregnancy rates has replaced conventional IVF and other types of micromanipulation as first-line therapy in couples with severe male factor infertility (PayneD1994, PalermoG1993\& Van
SteirteghemAC(1993). The high success rate of ICSI has led to extension of the technique to other patient populations for whom conventional IVF may be an option, including subfertile males and unexplained infertility (Aboulghar1996). However, the role of ICSI in men with mild sperm impairment and in men with normal sperm characteristics but low fertilization rates with conventional IVF treatment has not been well established (Shai E2004).

One of the major concerns in treating couples with moderate sperm impairment (moderate oligo \pm astheno \pm teratospermia) is the wide range of complete fertilization failure with conventional IVF treatment, reported as being present in $4 \%-50 \%$ of the couples. It is tempting to propose ICSI to those couples as a means of decreasing complete fertilization failure (VerheyenG1999, Aboulghar1996, FishelS2000 \& PlachotM2002). ICSI, however, is time consuming, expensive, and involves unresolved concerns regarding the short- and long-term outcomes of the conceived newborns (HansenM2002).

Due to these conflicting results, several groups have suggested that conventional IVF and ICSI should be performed on sibling oocytes (the IVF-ICSI split procedure) in patients with male subfertility to reduce the risk of complete fertilization failure (Pisarska1999, VerheyenG1999, VanSteireghem1993,PlachotM2002,

CalderonG1995\&Vander Westerlaken2006); others have suggested this technique in couples with unexplained infertility (HershlagA2002) or with previous unexplained fertilization failure (Fishel S2000, Van der Westerlaken2006 \& Benadiva1999).

We therefore conducted a study in which half the oocytes from couples with mild male factor infertility (mild oligo \pm astheno \pm teratospermia) were inseminated (IVF) and the other half microinjected (ICSI).

The purpose of the current study was to assess whether the performance of the IVF- 
ICSI split insemination method can improve fertilization rates and reduce the risk of fertilization failure in couples with mild male factor infertility.

\section{Materials and Methods}

\section{Patients:}

This prospective study included a total of 190 couples attending the in vitro fertilization (IVF) program of IVF Department (Enjab Hospital) between August 2007 and April 2010. The cases with subfertile semen parameters were selected to undergo an IVF cycle in which oocytes were divided without prejudice between conventional IVF and ICSI.

Mild male-factor subfertility was defined by the presence of at least one abnormal semen parameter, i.e., count of $5-20 \times 10^{6} / \mathrm{mL}$, motility of $25-40 \%$, or $20 \%-30 \%$ of spermatozoa classed as morphologically normal according to World Health Organization (1999) criteria and Kruger strict criteria (1986 \& 1988).

Patients were included in this study on the basis of previous diagnostic semen analyses and when, on the day of oocyte retrieval, their semen fulfilled the above criteria again. Women who had poor response $(<$ four retrieved oocytes) or prior enrolled in this study were excluded.

The study got approval from UAE ethical committee (UEC) and informed consent of patients was documented before being included in the study.

\section{Setting:}

This study was conducted at private IVF/ICSI units (Enjab Hospital for infertility and Gulf Medical College and Research Centre, Sharjah, UAE).

\section{Stimulation protocol:}

The women participating in this study followed a long $\mathrm{GnRH}$ agonist protocol that began with daily S.C injections of $0.1 \mathrm{mg}$ triptoreline (Ipsen pharma biotech, France) on Day 21 of the pre-stimulation cycle. The $\mathrm{GnRH}$ agonist was continued until the day of HCG administration. E2 levels less than $50 \mathrm{pg} / \mathrm{mL}$ on cycle day 3 and endometrial thickness less than $4 \mathrm{~mm}$ indicated downregulation. Gonadotropin was administered daily by S.C injection of follitropin beta (Puregon; Organon, Netherlands) in a dose of $200 \mathrm{IU} /$ day started from the third day of the cycle for five days after which the level was adjusted according to the associated ovarian response in order to stimulate follicular development. The resultant ovarian response was monitored by transvaginal ultrasound and serum E2 levels. In cases of less than three growing follicles on cycle day 14, treatment cycle was canceled. When three or more follicles reached a maximum diameter of $18 \mathrm{~mm}$, 10,000 IU of hCG (Pregnyl; Organon, Holland) was administered.

\section{Semen Preparation:}

Freshly ejaculated semen was allowed to liquefy. Volume was determined, concentration and percentage of motile spermatozoa were assessed in a Makler counting chamber, and the total number of motile spermatozoa was calculated. The semen sample was diluted 1:2 with Sperm preparation media (Medicult; Lucron, Milsbeek, the Netherlands) in a $12-\mathrm{mL}$ tube and was processed by centrifuge (1500-2000 $\mathrm{r} /$ minute for 10 minutes). The supernatant was removed, and the sperm pellet $(0.1-0.5$ $\mathrm{mL}$ ) was re-suspended in $1 \mathrm{~mL}$ of Sperm preparation media and processed by centrifuge (1500-2000 r/minute for 5 minutes). The supernatant was removed, and the sperm pellet $(0.1-0.5 \mathrm{~mL})$ was used. Volume, concentration, motility, and the total motile sperm count were re-determined after processing. The spermatozoa were kept at $37^{\circ} \mathrm{C}$ in a $\mathrm{CO} 2$ incubator until IVF or ICSI took place.

\section{Oocyte Retrieval and Preparation:}

Transvaginal oocyte retrieval was performed under general anesthesia by one operator (A.M) 34-36 $\mathrm{h}$ after $\mathrm{hCG}$ injection. The 
retrieved oocyte-cumulus complexes (OCCs) were pooled and washed in HEPESbuffered Earle's medium and then randomly transferred in groups of two to six OCCs (depending on the total number of OCCs retrieved) to droplets of $25-\mu \mathrm{L}$ of culture medium (universal IVF medium; Medicult) under mineral oil (Sigma, Brunswig Chemie, Amsterdam, the Netherlands) and then put into an incubator $\left(37^{\circ} \mathrm{C}, 5 \% \mathrm{CO} 2\right)$. Before injection or insemination, the OCCs were taken out of the incubator and randomly divided for ICSI or IVF insemination.

The OCCs that were assigned to ICSI were denuded of their surrounding cumulus cells both enzymatically and mechanically at 0-2 hours after retrieval. The maturation stage was checked, and the oocytes that had extruded a polar body were selected for injection. After injection, the oocytes were transferred to $25-\mu \mathrm{L}$ droplets of universal IVF medium, in which they were cultured individually.

The OCCs that were assigned to IVF kept their surrounding cumulus cells, and they were cultured individually in $25-\mu \mathrm{L}$ droplets of universal IVF medium. Each oocyte was inseminated with 75,000-150,000 motile spermatozoa (standard number is 75,000), 2-4 hours after oocyte retrieval, in a total volume of $25-30 \mu \mathrm{L}$.

\section{Assessment of Fertilization and cleavage:}

Sixteen to 18 hours later, the oocytes were inspected for normal, two-pronuclear fertilization. The rate of fertilization was calculated per OCC. Cleavage and embryo quality was evaluated at days 2 and 3 after oocyte retrieval. Embryos were assessed for their morphology and number of cells. Rapidcleavage embryos were defined as embryos sized at least four cells at 2 days after insemination and sized at least six cells at 3 days after insemination.

\section{Embryo Transfer and Pregnancy Testing:}

Embryo transfer took place 3 days after oocyte retrieval under ultrasonic guide. The highest quality embryos were transferred regardless of their method of insemination. Depending on the woman's age and the embryo quality, two to three embryos were transferred. Good-quality excess embryos were cryopreserved.

On the $14^{\text {th }}$ days of the transfer, a serum $\beta$-hCG test was performed to confirm pregnancy. Pregnancy was defined by an increasing $\beta$-hCG of $\geq 50 \mathrm{IU} / \mathrm{L}$ at 14 days after oocyte retrieval. Ongoing pregnancy was defined by the presence of a gestational sac with fetal heartbeat after 12 weeks of gestation.

\section{Data registration and Statistical analysis:}

The results were tabulated and statistically analyzed using a computer program SPSS (statistic a package for social science), version 15. The sample mean (X), standard deviation (SD), and standard error of the mean as well as the range were obtained for numerical variables. For non-numerical variables, the frequency, distribution and percentage were calculated. The student's ( $\mathrm{t}$ ) test was used to test the significance of the difference between 2 independent means. The Chi square test $\left(\mathrm{X}^{2}\right)$ was used to test whether the distribution of a certain phenomenon among two or more groups was equal or not.

\section{Results}

Two hundred and five patients undergoing IVF and eligible for our criteria were started the treatment in this study. Four patients with very poor response (less than 2 growing follicles on cycle day 14) and three patients with less than 4 retrieved oocytes were excluded. On the other hand, three patients were refused to participate and five patients were not meeting inclusion criteria. One hundred and ninety patients were randomized to undergo an IVF cycle in which oocytes were divided without prejudice between conventional IVF and ICSI. The flowchart of the patients included in the study is shown in figure 1 . 


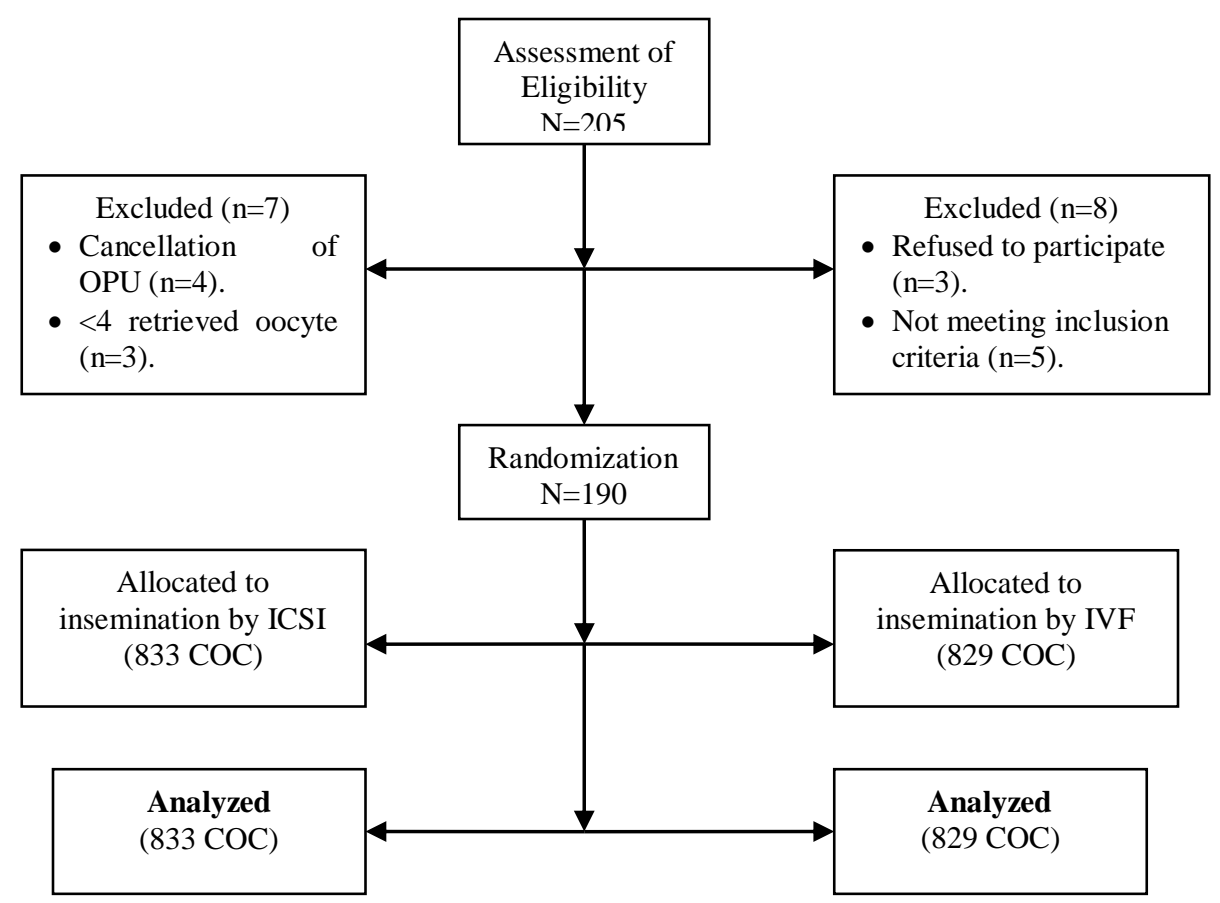

Figure 1: Flowchart of the patients included in this study.

Table 1: Collective data of oocytes inseminated by conventional IVF and ICSI in 190 patients with mild male factor infertility

\begin{tabular}{|l|l|l|l|}
\hline & $\begin{array}{l}\text { Oocytes retrieved } \\
(\mathrm{n}=1662)\end{array}$ & $\begin{array}{l}\text { Oocytes by ICSI } \\
(\mathrm{n}=833)\end{array}$ & $\begin{array}{l}\text { Oocytes by IVF } \\
(\mathrm{n}=829)\end{array}$ \\
\hline $\begin{array}{l}\text { Patients with fertilization by } \\
\text { IVF and ICSI }(\mathrm{n}=145)\end{array}$ & 1282 & $642(50.1 \%)$ & $640(49.9 \%)$ \\
\hline $\begin{array}{l}\text { Patients with fertilization only } \\
\text { by ICSI }(\mathrm{n}=41)\end{array}$ & 351 & $176(50.1 \%)$ & $175(49.9 \%)$ \\
\hline $\begin{array}{l}\text { Patients without fertilization } \\
\text { by ICSI and IVF }(\mathrm{n}=4)\end{array}$ & 29 & $15(51.7 \%)$ & $14(48.3 \%)$ \\
\hline
\end{tabular}

In 190 oocyte retrievals, 1,662 oocytes were collected and divided randomly without prejudice between conventional IVF and ICSI procedures. 833 oocytes were allocated to the ICSI procedure (746 of them were microinjected) and 829 oocytes were assigned to the conventional IVF procedure

$77.2 \%$ of retrieved oocytes (1282/1662) were fertilized by both IVF and ICSI, $21.1 \%$ of retrieved oocytes (351/1662) were fertilized only by ICSI and $1.7 \%$ of retrieved oocytes (29/1662) were not fertilized either by IVF 
On the other hand, $76.3 \%$ of patients (145/190) were fertilized by both IVF and ICSI, $21.6 \%$ of patients $(41 / 190)$ were fertilized only by ICSI while $2.1 \%$ of patients $(4 / 190)$ were not fertilized either by IVF or ICSI. These data are provided in Table 1.

Table 2: Sperm parameters of the 190 patients included in this study

\begin{tabular}{|c|c|c|c|}
\hline & $\begin{array}{l}\text { Concentration } \\
\left(\mathrm{X} 10^{6} \mathrm{ml}\right)\end{array}$ & $\begin{array}{l}\text { Motility percentage } \\
(\%)\end{array}$ & $\begin{array}{l}\text { Total motile count } \\
(\mathrm{X} \mathrm{10})\end{array}$ \\
\hline $\begin{array}{l}\text { Patients with fertilization by } \\
\text { IVF and ICSI }(n=145) \\
-\quad \text { Before Processing } \\
\text { - } \quad \text { After Processing }\end{array}$ & $\begin{array}{l}24.2 \pm 21.7 \\
31.6 \pm 25.4\end{array}$ & $\begin{array}{l}42.5 \pm 22.6 \\
59.7 \pm 29.4(\mathrm{c})\end{array}$ & $\begin{array}{l}28.5 \pm 24.9 \text { (a) } \\
8.6 \pm 4.8\end{array}$ \\
\hline $\begin{array}{l}\text { Patients with fertilization } \\
\text { only by ICSI ( } \mathrm{n}=41) \\
-\quad \text { Before Processing } \\
-\quad \text { After Processing }\end{array}$ & $\begin{array}{l}18.8 \pm 13.4 \\
25.7 \pm 19.2\end{array}$ & $\begin{array}{l}36.5 \pm 19.3 \\
43.4 \pm 22.6(d)\end{array}$ & $\begin{array}{l}20.4 \pm 15.7(b) \\
6.7 \pm 5.2\end{array}$ \\
\hline $\begin{array}{l}\text { Patients without fertilization } \\
\text { by ICSI and IVF }(n=4) \\
-\quad \text { Before Processing } \\
-\quad \text { After Processing }\end{array}$ & $\begin{array}{l}16.4 \pm 11.6 \\
21.5 \pm 13.8\end{array}$ & $\begin{array}{l}30.1 \pm 9.4 \\
39.6 \pm 12.9\end{array}$ & $\begin{array}{l}7.3 \pm 3.9 \\
4.9 \pm 3.6\end{array}$ \\
\hline
\end{tabular}

a,b: Significantly different from each other $(\mathrm{P}<0.05)$

c,d: Significantly different from each other $(\mathrm{P}<0.01)$

Sperm parameters before and after preparation in all patients are shown in Table 1. Significant differences were found in patients with fertilization by both IVF and ICSI compared with fertilization only by ICSI with regard to motility after preparation $(59.7 \pm 29.4 \%$ vs. $43.4 \pm 22.6$ $\% ; \mathrm{P}<0.05)$ and the mean total motile sperm count before preparation $\left(28.5 \pm 24.9 \times 10^{6}\right.$ vs. 20.4 $\pm 15.7 \times 10^{6}, \mathrm{P}<0.01$ ). For the four patients with no fertilization in both the IVF-treated and the ICSI-treated oocytes, the sperm parameters were not significantly different from those of the other two groups. 
Table 3: Outcome after conventional IVF and ICSI performed on sibling oocytes in 190 patients with mild male factor infertility

\begin{tabular}{|l|l|l|l|l|}
\hline & \multicolumn{2}{|l|}{ Fertilization } & \multicolumn{2}{l|}{ Type 1-2 Embryos } \\
\cline { 2 - 5 } & $\begin{array}{l}\text { ICSI fertilized } \\
(\mathrm{n}=531)\end{array}$ & $\begin{array}{l}\text { IVF fertilized } \\
(\mathrm{n}=367)\end{array}$ & $\begin{array}{l}\text { ICSI embryos } \\
(\mathrm{n}=(403)\end{array}$ & $\begin{array}{l}\text { IVF embryos } \\
(\mathrm{n}=207)\end{array}$ \\
\hline & $(63.7 \%)^{\mathbf{a}}$ & $\begin{array}{l}403 / 531 \\
(75.9 \%)^{\mathbf{c}}\end{array}$ & $\begin{array}{l}207 / 367 \\
(56.4 \%)^{\mathbf{d}}\end{array}$ \\
\hline $\begin{array}{l}\text { Patients with fertilization } \\
\text { by IVF and ICSI (n= 145) }\end{array}$ & $\begin{array}{l}423 / 642 \\
(65.9 \%)\end{array}$ & $367 / 640(57.3 \%)$ & $\begin{array}{l}327 / 423 \\
(77.3 \%)^{\mathbf{e}}\end{array}$ & $\begin{array}{l}\mathbf{2} \\
(56.4 \%)^{\mathbf{f}}\end{array}$ \\
\hline $\begin{array}{l}\text { Patients with fertilization } \\
\text { only by ICSI (n= 41) }\end{array}$ & $\begin{array}{l}108 / 176 \\
(61.4 \%)\end{array}$ & $\begin{array}{l}0 / 175 \\
(0 \%)\end{array}$ & $76 / 108(70.4 \%)$ & NA \\
\hline $\begin{array}{l}\text { Patients without } \\
\text { fertilization by ICSI and }\end{array}$ & $\begin{array}{l}0 / 15 \\
(0 \%)\end{array}$ & $\begin{array}{l}0 / 14 \\
(0 \%)\end{array}$ & NA & NA \\
\hline
\end{tabular}

a,b Significantly different from each other $(\mathrm{P}<.05)$. $c, d$ Significantly different from each other $(\mathrm{P}<.05)$. e,f Significantly different from each other $(\mathrm{P}<.01)$. $\mathrm{NA}=$ not applicable.

Among 145 of the 190 treated couples, fertilization occurred both after ICSI (423/642 oocytes; $65.9 \%$ ) and after IVF (367/640 oocytes; 57.3\%), whereas among 41 of the 190 couples, fertilization was observed only after ICSI (108/176 oocytes; $61.4 \%)$ and not after IVF. In four couples, there was no fertilization after ICSI (0/15 oocytes) and IVF (0/14 oocytes). All these patients had mature oocytes and no morphological abnormalities of the oocytes were observed.

In the current study, fertilization rate was significantly higher in oocytes injected by ICSI (63.7\%) compared to oocyte inseminated by IVF (44.3\%). In patients fertilized by both IVF and ICSI, although ICSI fertilization rate was higher $(65.9 \%)$ than IVF fertilization rate $(57.3 \%)$, yet there is no significant differences were observed. These data are provided in Table 2.

High grade embryos were significantly higher in oocytes injected by ICSI (75.9\%) compared to oocyte inseminated by IVF (56.4\%). Also, significantly more type 1 and 2 embryos developed after ICSI compared with IVF $(77.3 \%$ vs. $56.4 \%)$ in the group of patients with fertilization after both IVF and ICSI. There was no difference in embryo quality between ICSI embryos developed in cycles with and without fertilization in IVF $(77.3 \%$ vs. $70.4 \%)$. These results also are given in Table 3.

Table 4: Embryo transfer, pregnancy rate, and implantation rate (178 patients).

\begin{tabular}{|l|l|l|l|l|l|}
\hline & \multicolumn{3}{|l|}{$\begin{array}{l}\text { Patients with fertilization by IVF and ICSI } \\
(\mathrm{n}=139)^{\mathbf{a}}\end{array}$} & $\begin{array}{l}\text { Patients with fertilization } \\
\text { only by ICSI }(\mathrm{n}=39)^{\mathbf{b}}\end{array}$ \\
\hline & IVF & IVF+ICSI & ICSI & ICSI & IVF \\
\hline Transfers & $32(23.0 \%)$ & $46(33.1 \%)$ & $61(43.9 \%)$ & 39 & 0 \\
\hline Pregnancies & $12(37.5 \%)$ & $20(43.5 \%)$ & $28(45.9 \%)$ & $18(46.2 \%)$ & 0 \\
\hline Ongoing pregnancies & $11(34.4 \%)$ & $19(41.3 \%)$ & $26(42.6 \%)$ & $16(41.0 \%)$ & 0 \\
\hline
\end{tabular}


a

In 6 patients, there was no transfer because of ovarian hyperstimulation syndrome and unsuitable embryos for transfer.

b

In 2 patients there was no transfer because of ovarian hyperstimulation syndrome .

In 12 of the 190 patients there was no transfer; in 5 patients, because of ovarian hyperstimulation syndrome, in 4 patients because of fertilization failure and in 3 patients, because there was no suitable embryos.

Overall, more ICSI embryos were transferred compared with the case of IVF embryos (335 vs. 163).

In patients with fertilization by IVF and ICSI $(n=139), 226$ ICSI embryos and 163 IVF embryos were transferred: 32 transfers of only IVF embryos (23.0\%), 46 transfers of a mixture of IVF and ICSI embryos (33.1\%), and 61 transfers of only ICSI embryos (43.9\%).

In total, 78 patients became pregnant: 60 in the group with fertilization after both IVF and ICSI (42.3\% per transfer) and 18 in the group with fertilization only after ICSI (46.2\% per transfer) and). No significant differences were found with regard to pregnancy rates and ongoing pregnancies between the three groups of embryo transfers (IVF, IVF-ICSI, and ICSI). These data are given in Table 4.

\section{Discussion}

Reports of higher fertilization rates after ICSI suggesting that this technique may be better than conventional methods for all couples seeking IVF have led to controversy for a long time. However, among other concerns, current knowledge of ICSI as an outcome procedure does not provide the confidence to use this process in all cases of IVF for the time being, even if ICSI offers a higher incidence of fertilization, maximizes the number of embryos, and minimizes the risk of complete failure of fertilization (Fishel S 2000 \& Van Rumste MM 2004).

Comparing the clinical outcomes obtained from IVF vs. ICSI is difficult because each treatment deals with different infertility indications. Reports regarding the outcomes of IVF vs. ICSI are controversial and complicated by different inclusion criteria for ICSI. Hsu et al. (1999) reported that IVF-derived day-3 embryos had better cleavage rates and morphology scores than did ICSI-derived day-3 embryos in consecutive couples undergoing ART therapy. Dumoulin et al. (2000) demonstrated that embryos obtained after
ICSI had a decreased potential to develop into blastocysts. Chang et al. (2000), Lan et al (2001) and Yoeli et al (2008) also revealed that embryo quality does not seem to be influenced by the mode of fertilization (IVF or ICSI). Yu-Che et al (2010) assumed that embryo quality depends on intrinsic factors of the gametes involved, rather than on the fertilization process per se.

In patients with borderline semen, the decision to choose either conventional IVF or ICSI is critical because the chance of total fertilization failure after a conventional IVF or of performing an unnecessary ICSI procedure is hard to predict. The question is how to discriminate between patients who do and do not need ICSI to fertilize. In the literature, there is no answer to this question. Van der Westerlaken et al (2006) concluded that in patients with subfertile semen, the treatment of sibling oocytes with both IVF and ICSI remains the optimal tool to prevent total fertilization failure after conventional IVF

Plachot et al (2002) in their study confirms that performing conventional IVF and ICSI 
in sibling oocytes in the first cycle for couples with borderline semen quality decreases the risk of transfer cancellation over that for conventional IVF alone. In addition, it is also an excellent test of sperm fertilizing ability, to be used as a guideline for the management of possible future cycles.

Similar studies comparing IVF and ICSI in sibling oocytes from couples with borderline semen quality have been reported (PisarskaMD 1999, VerheyenG 1999, Aboulghar1996, Plachot M2002, Calderon G1995 \& Van der Westerlken2006). All concluded that this practice prevented the cancellation of embryo transfer due to complete fertilization failure after conventional IVF (which occurs in $25-50 \%$ of the cycles) and the cycles with fertilization after both IVF and ICSI show similar fertilization rates of the IVF- and ICSI-treated oocytes. Indeed, the overall fertilization rate was higher after ICSI (50$63 \%$ depending on the study) than after IVF (18-23\%).

In case of male subfertility, the ICSI treatment results in significant higher fertilization rate per oocyte compared with conventional IVF treatment in this study $(63.7 \%$ vs. $44.3 \%)$. However, when the fertilization percentage is calculated per patient, three groups of patients can be discriminated: one with fertilization after both conventional IVF and ICSI, one with fertilization only after ICSI, and one without fertilization after either conventional IVF or ICSI. In the first group, although ICSI fertilization rate was higher $(65.9 \%)$ than IVF fertilization rate $(57.3 \%)$, yet there is no significant differences were observed.

These results are in agreement with Michael et al (2003), Shai et al (2004), Hackett et al (2005), Gvakharia et al (2005), and Van der Westerlaken et al (2006).

Michael et al (2003) in their retrospective study to assess the value of split ICSI/Insemination in mild male factor infertility or in case of complete fertilization failure with normal semen parameters, concludes that the routine use of this method will maximize fertilization rates and the yield of good-quality embryos.

Shai et al (2004) suggested that, in their retrospective study, implementation of split ICSI in couples with mild male factor infertility could improve fertilization rates and decrease the risk of complete fertilization failure but possibly may be associated with a lower number of early cleaving embryos. On the other hand, they did not encourage performing this procedure in infertile couples with normal sperm characteristics.

Hackett et al (2005) in their retrospective analysis to determine the effect of insemination method on embryo development within a cohort of eggs, they found that the fertilization rate, determined by the number of 2 pronuclear zygotes on day1 divided by the number of eggs inseminated, was

significantly different (ICSI 68\%; IVF $47 \%$ ). Significance was lost however when the fertilization rate for the ICSI group was calculated by the total number of eggs available rather than by those that were mature (ICSI 53\%; IVF 47\%). The occurrence of failed fertilization was significantly different in the two groups (ICSI 2.5\%; IVF $19.2 \%$ p $<0.0001$ ). Finally, they suggest that in patient with infertility of unknown etiology a split IVF/ICSI cycle can help reduce failed fertilization.

Gvakharia et al (2005) in their retrospective study to analyze laboratory and clinical outcome indicators of split ICSI/Insemination in mild male factor concludes that Split ICSI is a reasonable option for patients with sub-optimal sperm parameters and also for patients who wish to minimize the risk of fertilization failure in IVF. In $10 \%$ of patients, the split ICSI procedure avoided total failure of fertilization and supplied embryos for transfer. 
Van der Westerlaken et al (2006) in their randomized study, they found in the patients fertilized after both conventional IVF and ICSI, the fertilization percentages are same and they suggest an all-or-nothing effect with regard to the fertilization capacity in conventional IVF. Patients with fertilization after conventional IVF apparently do not need to be treated with ICSI and once fertilization has been established, there is no difference in developmental competence between IVF and ICSI treatment.

In contrast to our finding, Hershlag et al (2002) found no improvement in fertilization rates after ICSI in cases with borderline semen characteristics. In their study, however, borderline sperm was defined as sperm with $20 \%-30 \%$ motility, which is nearly the same as the definition we used in our study: motility of $25 \%-40 \%$. However, they did not pay attention to other sperm parameters as count and morphology which may partly explain the difference in our findings.

With regard to embryo quality, we found significantly higher quality embryos after ICSI compared with IVF (75.9\% vs $56.4 \%)$. Also, we found significantly higher quality embryos after ICSI $(77.3 \%)$ compared with IVF $(56.4 \%)$ in patients who became fertilized both after IVF and after ICSI. This difference did not reach significance when ICSI embryos from patients who became fertilized only after ICSI (70.4\%) were compared with the IVF-fertilized embryos (77.3\%).

This is in agreement with Michael et al (2003) and van der Westerlaken et al (2006) but not in agreement with other studies that did not find differences in embryo quality between IVF and ICSI (Pisarska MD 1999, VerheyenG 1999, Plachot M2002 \& Tournaye H2002).
Whether it is the technique (IVF or ICSI) that is responsible for this observation is not clear. Patient variation as well as differences in sperm characteristics can be excluded as a possible explanation because sibling oocytes and the same semen sample have been used to establish fertilization. It might be that the ICSI embryos develop faster than the IVF embryos. This is a known phenomenon inherent to the ICSI technique (Nagy Z1998). It might also be that exposure of the IVF embryos to large numbers of spermatozoa, creating suboptimal culture conditions, affects embryo quality negatively. This negative effect might be avoided by using the short insemination method in conventional IVF (Quinn P1998 \& Menezo Y2000). The fact that more ICSI embryos are transferred than IVF embryos (table4) can be explained by the fact that there are more ICSI embryos available and that the ICSI embryos are of a higher quality.

The embryos in the current study were transferred based on embryo quality regardless of their origin from ICSI or conventional IVF. So the IVF and ICSI embryos that were transferred were of similar quality. This explains the similar (ongoing) pregnancy rates between IVF and ICSI treatment (table 4).

In conclusion, according to our findings, implementation of ICSI in couples with mild male factor infertility could improve fertilization rates and decrease the risk of complete fertilization failure. Additionally, split ICSI procedure provides valuable clinical information about fertilization potential for the couple and unnecessary use of ICSI procedure can be avoided in future cycles for patients who have achieved good fertilization in both IVF and ICSI. 


\section{References}

-Aboulghar, M.A., Mansour, R.T., Serour, G.I., Amin, Y.M. and Kamal, A. (1996)Prospective controlled randomized study of in vitro fertilization versus intracytoplasmic sperm injection in the treatment of tubal factor infertility with normal semen parameters. Fertil. Steril.,;66,753-756.

-Benadiva, C.A., Nulsen, J., Siano, L., Jennings, J., Bet Givargis, H. and Maier, D. (1999)Intracytoplasmic sperm injection overcomes previous fertilization failure with conventional in vitro fertilization. Fertil. Steril., ;72, 1041-1044.

-Calderon G, Belil I, Aran B, Veiga A, Gil Y, Boada M, et al.(1995) Intracytoplasmic sperm injection versus conventional in-vitro fertilization (first results). Hum Reprod. ;10:2835-2839.

- Chang SY, Lien KC, Huang FJ, Kung FT, Tsai MY.(2000) Comparable clinical outcomes of tubal embryo transfer for oligoasthenoteratozoospermia treated with intracytoplasmic sperm injection and for female infertility treated with in vitro fertilization. Chang Gung Med J. ;23:253-259.

-Dumoulin JCM, Coonen E， Bras M, van Wissen L, Ignoul-Vanvuchelen R, BergersJansen JM, et al.(2000) Comparison of in-vitro development of embryos originating from either conventional IVF or ICSI (Hum. Reprod.) ;15:402-409.

-Edirisinghe WR,Murch A,Junk S,Yovich JL (1997) Cytogenetic abnormalities of unfertilized oocytes generated from in-vitro fertilization and intracytoplasmic sperm injection (a double-blind study). Hum Reprod. ;12:2784-2791.

-Fishel S, Aslam I, Lisi F, Rinaldi L, Timson J, Jacobson M, et al.(2000) Should ICSI be the treatment of choice for all cases of in-vitro conception?. Hum Reprod. ;15:12781283.

-Guzick DS, Overstreet JW, Factor-Litvak P, Brazil CK, Nakajima ST, Coutifaris C, et al(2001) Sperm morphology, motility, and concentration in fertile and infertile men. N Engl JMed.;349:1388-1393.

-Gvakharia M., Z.L. Beri, V.L. Baker, G.D. Adamson.(2005) Outcomes of ICSI and Conventional Insemination of Sibling Oocytes.
ASRM;84;S1:370.

Hackett R.J., J.R. Trimarchi, A. Blazar,

-K. Pagidas, D.L. Keefe.(2005) Embryos From ICSI Do Not Develop Differently Than Those From IVF Within Split IVF/ICSI Cycles. ASRM,84;S1:455.

-Hansen M, Kurinczuk JJ, Bower C, Webb S.(2002) 456 major risk of birth defects after intracytoplasmic sperm injection and in vitro fertilization. N Engl J Med. ;346:725-730.

-Hershlag A, Paine T, Kvapil G, Feng H, Napolitano B(2002) In vitro fertilizationintracytoplasmic sperm injection split: an insemination method to prevent fertilization failure. Fertil Steril .;7:229-232.

-Hsu MI, $\quad$ Mayer J, $\quad \begin{gathered}\text { Aronshon M, } \\ \text { Lanzendorf S, }\end{gathered}$ et al.(1999) Embryo implantation in in vitro fertilization and intracytoplasmic sperm injection: impact of cleavage status, morphology grade, and number of embryos transferred. Fertil Steril.;72:679-685.

-Kruger TF, Menkveld R, Stander FS, Lombard CJ, Van der merwe JP, Van Jyl JA.(1986) Sperm morphologic features as a prognostic factor in in-vitro fertilization. Fertil Steril46:1118-1123.

-Kruger TF, Acosta AA, Simmons KF, Swanson RJ, Matta JF, Oehninger S.(1988) Predictive value of abnormal sperm morphology in in vitro fertilization. Fertil Steril.;49:112-117. -Lan KC, Chang SY, Huang FJ, Kung FT, Tsai MY.(2004) In vitro fertilization versus intracytoplasmic sperm injection in the embryo transfer model: a comparison of outcomes. Taiwan J Obstet Gynecol.;40:1-7.

- Liu DY, Baker HWG(2000) Defective spermzona pellucida interaction (a major cause of failure of fertilization in clinical in-vitro fertilization). Hum Reprod.;15:702-708.

-Liu DY， Baker HWG(2002) Evaluation and assessment of semen for IVF/ICSI. Asian J Androl.;4:281-285.

-Mahutte NG, Arici A.(2003) Failed fertilization (is it predictable?). Curr Opin Obstet Gynecol.;15:211-218.

-Menezo Y， Barak Y.(2000) Comparison of day-2 embryos obtained either from ICSI or resulting from short insemination IVF (influence of maternal age). Hum Reprod.;15:1776-1778. 
-.Michael P. Steinkampf, Karen R. Hammond, Phillip A. Kretzer.(2003) Value of split intracytoplasmic sperm injection/insemination for mild male factor infertility. ASRM, ;80;S3:206.

-Nagy Z , Janssenswillen C , Janssens R , de Vos A , Staessen C , Van de Velde H , et al.(1998) Timing of oocyte activation, pronucleus formation and cleavage in humans after intracytoplasmic sperm injection (ICSI) with testicular spermatozoa and after ICSI or invitro fertilization on sibling oocytes with ejaculated spermatozoa. Hum Reprod.;13:16061612.

-Palermo G, Joris H, Derde MP, Camus M, Devroey P, Van Steirteghem AC(1993) Sperm characteristics and outcome of human assisted fertilization by subzonal insemination and intracytoplasmic sperm injection. Fertil Steril.;59:826-835.

-Payne D, Flaherty SP, Jeffrey R, Warnes GM, Matthews CD(1994) Successful treatment of severe male factor infertility in 100 consecutive cycles using intracytoplasmic sperm injection. Hum Reprod 9:2051-2057.

-Plachot M, Belaisch-Allart J, Mayenga JM, Chouraqui A, Tesquier L, Serkine AM.(2002) Outcome of conventional IVF and ICSI on sibling oocytes in mild male factor infertility. Hum Reprod . ;17:362-369. 96-10.

- Pisarska MD, Casson PR, Cisneros PL, Lamb DJ, Lipshultz LI, Buster JE, et al(1999) Fertilization after standard in vitro fertilization versus intracytoplasmic sperm injection in subfertile males using sibling oocytes. Fertil Steril.;71:627-632.

-Quinn P, Lydic ML, Ho M.(1998) Confirmation of the beneficial effects of brief coincubation of gametes in human in vitro fertilization. Fertil Steril. ;69:399-402.

-Shai E Elizur, M.D., Jacob Levron, M.D., Daniel S Seidman, M.D., Salim Kees, M.D., David Levran, M.D., Jehoshua Dor, M.D.(2004)Conventional in vitro fertilization versus intracytoplasmic sperm injection for sibling oocytes in couples with mild oligoteratoasthenozoospermia and couples with normal sperm. ASRM,;82;1: 241-243.

-Tournaye H, Verheyen G, Albano C, Camus M, Van Landuyt L, Devroey P, et al.(2002) Intracytoplasmic sperm injection versus in vitro fertilization (a randomized controlled trial and a meta-analysis of the literature). Fertil Steril. ;78:1030-1037.
-Van der Westerlaken Lucette, M.Sc., Nico Naaktgeboren, Ph.D., Harjo Verburg, M.D., Sandra Dieben, M.D., Frans M. Helmerhorst, Ph.D.(2006) Conventional in vitro fertilization versus intracytoplasmic sperm injection in patients with borderline semen: a randomized study using sibling oocytes. ASRM, ;85;2:395-400.

-Va Rumste MM, Evers JL, Farquhar CM.(2004) ICSI versus conventional techniques for oocyte insemination during IVF in patients with non-male factor subfertility: a Cochrane review. Hum Reprod. ;19:223-227.

-Van Steirteghem AC, Liu J, Joris H, Nagy Z, Janssenswillen C, Tournaye H, et al(1993)

Higher success rate by intracytoplasmic sperm injection than by subzonal insemination (report of a second series of 300 consecutive treatment cycles). Hum Reprod. ;8:1055-1060.

-Verheyen G, Tournaye H, Staessen C, De Vos A, Vandervorst M, Van Steirteghem A( 1999)Controlled comparison of conventional invitro fertilization and intracytoplasmic sperm injection in patients with asthenozoospermia . Hum Reprod.;14:2313-2319.

-Wall MB, Marks K, Smith TA, Gearon CM, Muggleton-Harris AL(1996) Cytogenetic and fluorescent in-situ hybridization chromosomal studies on in-vitro fertilized and Intracytoplasmic sperm injected "failed-fertilized" human oocytes. HumReprod 11:2230-2238.

-world Health Organization(1999) WHO laboratory manual for the examination of human semen and sperm-cervical mucus interac 5. Liu DY，Baker HWG(2000) Defective spermzona pellucida interaction (a major cause of failure of fertilization in clinical in-vitro fertilization). Hum Reprod.;15:702-708tion. 4th ed. Cambridge, England: Cambridge University Press.

- Yoeli R, Orvieto R, Ashkenazi J, Shelef M, Ben-Rafael Z, Bar-Hava I.(2008) Comparison of embryo quality between intracytoplasmic sperm injection and in vitro fertilization in sibling oocytes. J Assist Reprod Genet.;25:2328.

-Yu-Che Ou, M.D., Kuo-Chung Lan, M.D., Fu-Jen Huang, M.D., Fu-Tsai Kung, M.D., Ting-Hsun Lan, M.D., Shiuh Young Chang, M.D.(2010) Comparison of in vitro fertilization versus intracytoplasmic sperm injection in extremely low oocyte retrieval cycles. ASRM, ;93;1:0. 


\title{
تقيم الحيوانات المنوية من الزوج العقيم فى حالة ندرتها بين الحقن المجهرى لهـا داخل بويضـات

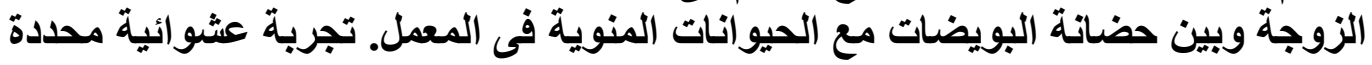

\author{
أشرف معوض هناء ابورية محمد عبد الظاهر محمد فرحات محمود شعير

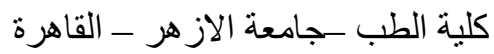

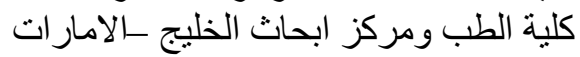 \\ مستشفى انجاب للعقم- الامارات
}

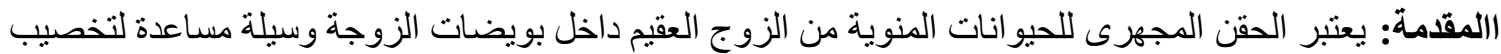

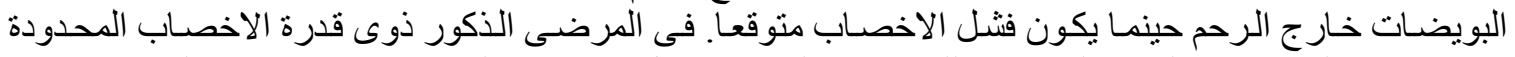

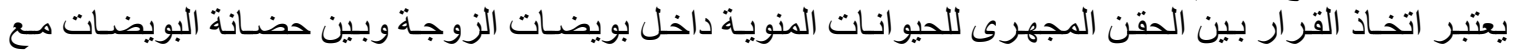

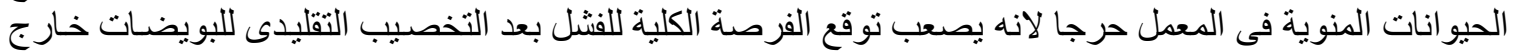

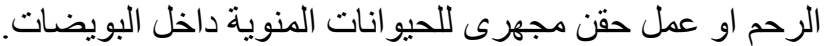

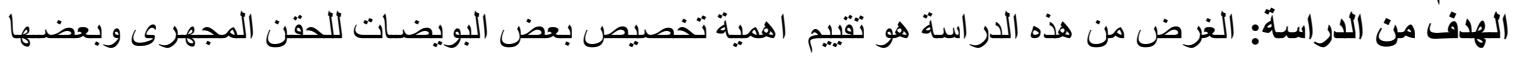

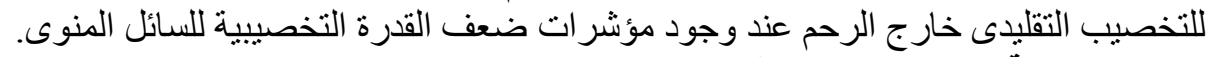
تصميم الدراسة: هذه در اسة مستقبلية.

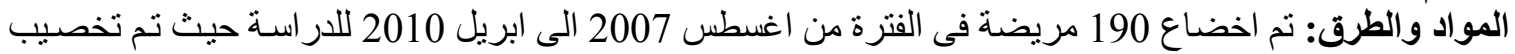

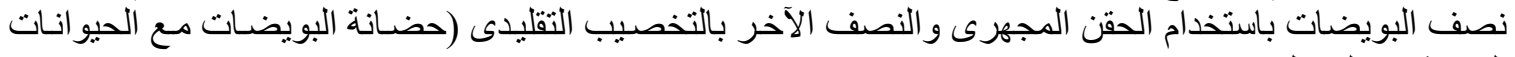
المنوية فى المعمل).

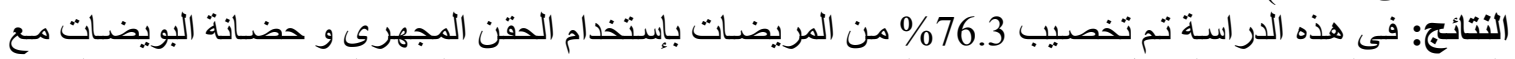

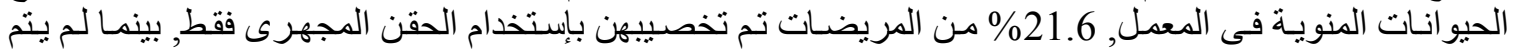

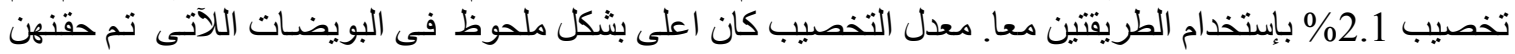

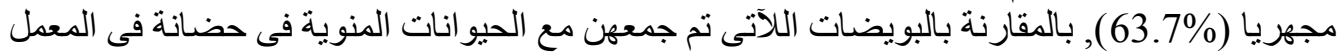

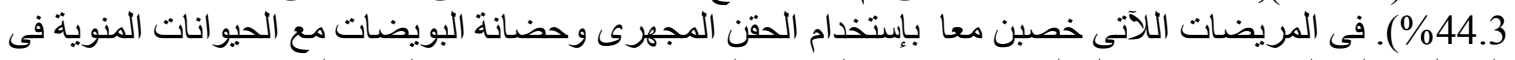

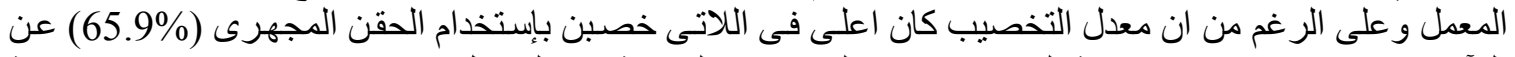

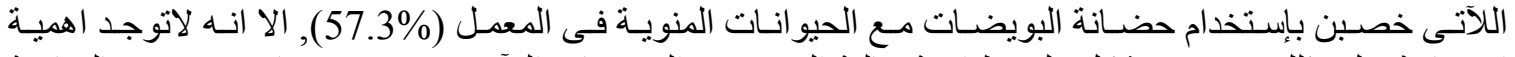

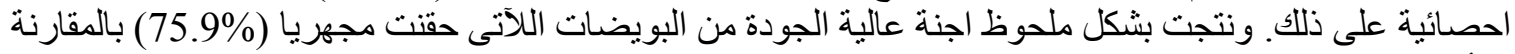

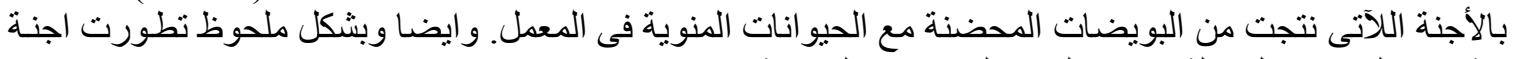

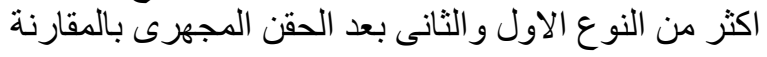

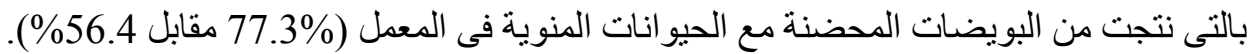

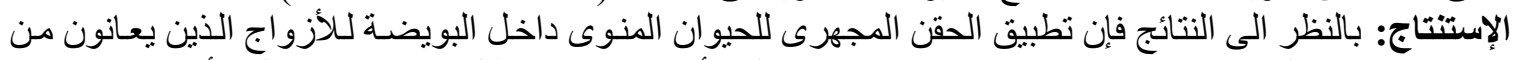

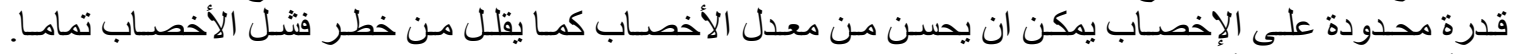

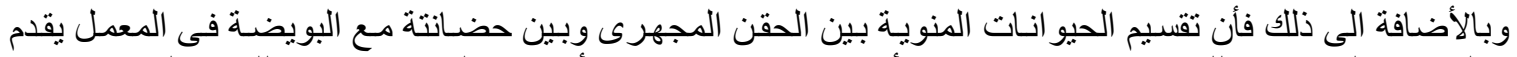

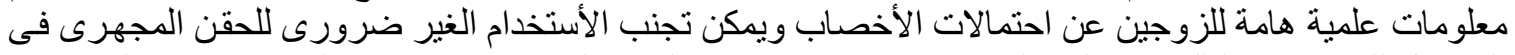

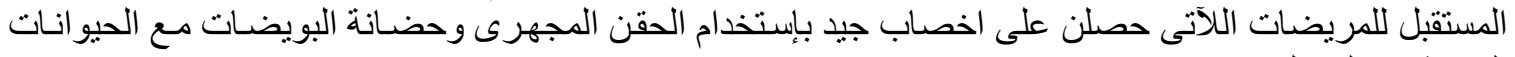
المنوية فى المعمل معا. 